\title{
Pre-eclampsia/eclampsia in twin pregnancies
}

\author{
A. MCFARLANE and J. S. SCOTT \\ From the Department of Obstetrics and Gynaecology (Leeds Maternity Hospital), University of Leeds, 17 Springfield \\ Mount, Leeds LS2 9NG
}

\begin{abstract}
Summary. A study of 1045 twin gestations with regard to known or likely zygosity and the incidence of pre-eclampsia/eclampsia failed to reveal differences between known dizygous twins and like-sex 'presumed' and 'estimated' monozygous twins except in the 'estimated' data for multigravidae. There was a threefold increase in the incidence for twins as opposed to singleton pregnancies. These results are discussed in relation to increased conceptus-mother antigenic differences. It is suggested that the risk of gestosis in twin pregnancy involves more than a summation of that operating in two singleton pregnancies.
\end{abstract}

It has been suggested that genetic incompatibility between mother and fetus may be a factor in the aetiology of pre-eclampsia (Penrose, 1946; Kalmus, 1946; Platt, Stewart, and Emery, 1958). Epidemiological evidence has been provided by Stevenson $e t$ al (1971) in a study of consanguineous marriages in the Middle East. They also recorded twin data which pointed to a higher incidence of toxaemia in unlike-sex as opposed to like-sex twin pregnancies. As all unlike-sex twins are dizygous $(\mathrm{DZ})$ and a large proportion of like-sex twins are monozygous (MZ), antigenic differences between mother and conceptus would be more likely to be expressed in unlike-sex twin pregnancies. Stevenson et al considered that the higher incidence with unlike-sex twins could be attributed to this. They extrapolated their data on the basis of Weinberg's hypothesis involving the assumption that for every pair of unlike-sex $\mathrm{DZ}$ twins there is one like-sex $D Z$ pair. The estimated differences in the incidence of pre-eclampsia based on this were significant at $P$ values of $<0.05$ to 0.001 , the syndrome being commoner in the presumed DZ cases. They regarded these findings as 'suggestive'.

This report concerns a review of twin deliveries in one hospital with regard to zygosity and the incidence of late pregnancy hypertension.

\section{Method}

The records of twin gestations from 1953 to 1973 inclusive at Leeds Maternity Hospital were studied.

Received 8 July 1975.
Pregnancies were classified according to definitions given below.

\section{A. Hypertension status}

(1) Mild pre-eclampsia-a basal blood pressure of 120/ $80 \mathrm{~mm} \mathrm{Hg}$ or less recorded before 24 weeks' gestation, followed by a rise to $140 / 90 \mathrm{~mm} \mathrm{Hg}$ or more on at least two occasions recorded in the antenatal ward, clinic readings being discounted, together with oedema and albuminuria of $<0.1 \mathrm{~g}$ per litre, if present.

(2a) Severe pre-eclampsia - as (1), with persistent albuminuria in late pregnancy of at least $0.1 \mathrm{~g}$ per litre in an uncontaminated urine.

(2b) Eclampsia - a typical fit occurring before, during, or within 24 hours of delivery associated with the signs as in (1) or (2a).

(3) Chronic hypertensive disease-a blood pressure of over $120 / 80 \mathrm{~mm} \mathrm{Hg}$ which had been high before 24 weeks.

(4) Unclassified 'toxaemia'-hypertension in late pregnancy confirmed in the antenatal ward but not otherwise fulfilling the criteria for pre-eclampsia or chronic hypertensive disease.

(5) Normal-all other cases.

\section{B. Parity status}

Cases were divided into nulliparous (no previous pregnancy of 28 weeks or more) and multiparous (one or more previous pregnancies of 28 weeks or more).

\section{Zygosity status}

Zygosity was classified as follows:

(a) Certainly $D Z$-all unlike-sex twins.

(b) Presumed $M Z$-like-sex twins with placentae recorded as monochorionic. 
(c) 'Estimated' $M Z$-total like-sex twins less number of unlike-sex twins (Weinberg's differential method).

To calculate the likely incidence of various categories of hypertensive disease under 'estimated' $M Z$, the number occurring in the certainly $\mathrm{DZ}$ category was subtracted from that in like-sex twins.

\section{Singleton comparison}

To compare the incidence of pre-eclampsia in twin and singleton pregnancies a matched-pair comparison was done. Twin records from every third year were matched against the next appropriate singleton record, excluding anencephalics. Matching covered the following particulars:

(a) Age-to within 5 years.

(b) Parity-primigravidae, previous abortions only, multiparae, grande multiparae ( 5 or more previous deliveries).

(c) Gestation length-30 weeks or less, 31 to 35 weeks, 36 weeks or more.

(d) Sex of child-in the case of unlike-sex twins alternate male and female singletons were taken.

\section{Results}

The results of the comparative analyses on different forms of hypertension and the different categories of twin pregnancies are shown in Table I.
None of the observed values indicates a significant difference between the 'certainly DZ' twins and other groups in relation to pre-eclampsia/eclampsia incidence. The 'estimated $M Z$ ' values give a lower figure for multiparae significant at the $5 \%$ level but in primigravidae the difference is reversed and when primigravidae and multigravidae are considered together there is no significant difference. Table II gives the details of the matched pair comparison between twins and singletons, indicating an approximate threefold increase in incidence of pre-eclampsia/eclampsia in twin as opposed to singleton pregnancies.

\section{Discussion}

With the exception of the data on 'estimated' $M Z$ multiparous twin pregnancies, these results suggest there is no difference in the incidence of preeclampsia/eclampsai between $\mathrm{DZ}$ and $\mathrm{MZ}$ pregnancies in this population. The exception was mainly contributed to by cases of mild pre-eclampsia and, as Adams and MacGillivray (1961) have pointed out, some such cases may not have true pre-eclampsia. Furthermore, in multiparous patients the diagnosis of pre-eclampsia is less accurate than in primigravidae. McCartney (1964), for example, using renal

TABLE I

INCIDENCE OF HYPERTENSIVE DISEASE AND TWIN CATEGORY

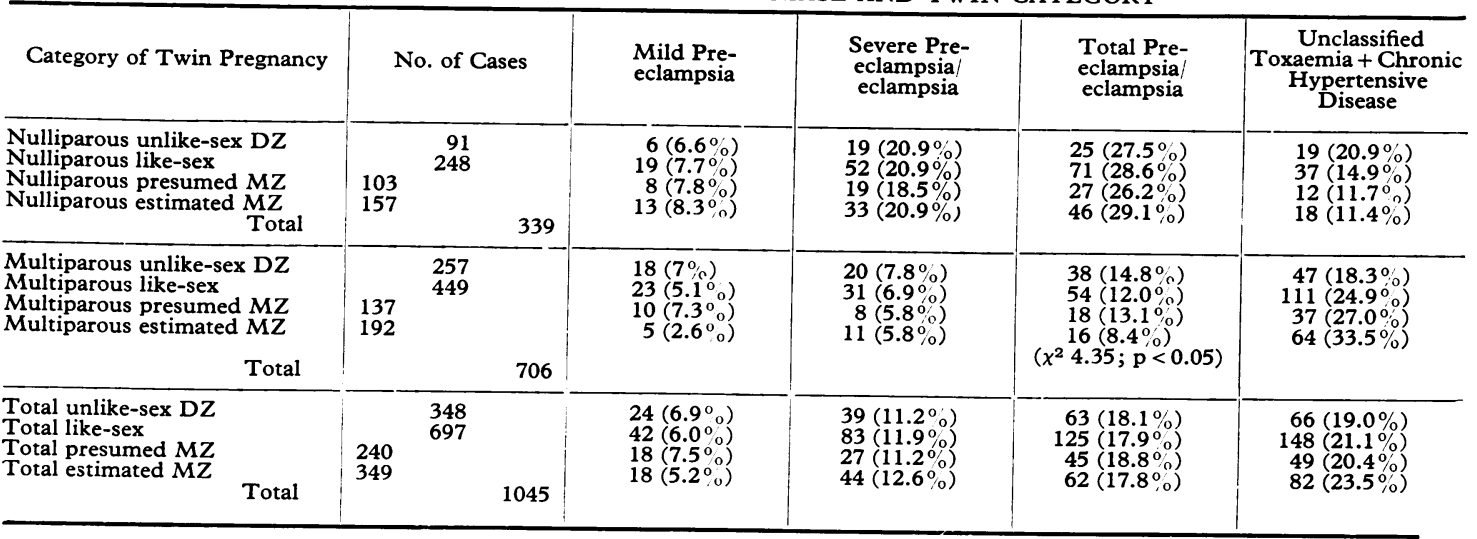

TABLE II

SINGLETON COMPARISON (MATCHED PAIRS)

\begin{tabular}{|c|c|c|c|c|c|c|}
\hline & & & $\begin{array}{l}\text { Mild Pre- } \\
\text { eclampsia }\end{array}$ & $\begin{array}{l}\text { Severe Pre- } \\
\text { eclampsia/ } \\
\text { eclampsia }\end{array}$ & Total Pre-eclampsia & $\begin{array}{c}\text { Unclassified Toxaemia } \\
\text { + Chronic Hypertensive } \\
\text { Disease }\end{array}$ \\
\hline (a) All maturities & $\begin{array}{l}\text { Twins } \\
\text { Singletons }\end{array}$ & $\begin{array}{l}337 \\
337\end{array}$ & $\begin{array}{l}19(5.6 \%) \\
10(3.0 \%)\end{array}$ & $\begin{array}{l}43(12.8 \%) \\
12(3.6 \%)\end{array}$ & $\begin{array}{c}62(18.4 \%) \\
22(6.5 \%) \\
\chi^{2}=21.7 ; \mathrm{p}=<0.001\end{array}$ & $\begin{array}{l}74(21.9 \%) \\
55(16.3 \%)\end{array}$ \\
\hline (b) 36 weeks or more & $\begin{array}{l}\text { Twins } \\
\text { Singletons }\end{array}$ & $\begin{array}{l}266 \\
266\end{array}$ & $\begin{array}{r}15(5.6 \%) \\
7(2.6 \%)\end{array}$ & $\begin{array}{c}37(13.9 \%) \\
6(2.3 \%)\end{array}$ & $\begin{array}{c}52(19.6 \%) \\
13(4.9 \%) \\
\left(\chi^{2}=26.5 ; \mathrm{p}=<0.001\right)\end{array}$ & $\begin{array}{l}66(24.8 \%) \\
49(18.4 \%)\end{array}$ \\
\hline
\end{tabular}


biopsies found typical histological lesions in $71 \%$ primigravidae but in only $14 \%$ multiparae in whom a clinical diagnosis of pre-eclampsia had been made. Furthermore, though Weinberg's differential method of estimating the proportion of $\mathrm{MZ}$ twins allows inclusion of all twins in the analysis it involves subtractions from the observed numbers of like-sexed twins, with the risk of distortion from random variations. It seems likely that the observed differences in pre-eclampsia in 'estimated' $M Z$ twin pregnancies in multiparae were attributable to factors such as these.

Accepting that our results do not show any difference between $D Z$ and $M Z$ twins with regard to pre-eclampsia, the question arises as to why they differ from those of Stevenson et al (1971). There are several factors that might affect the relative incidence of pre-eclampsia in $\mathrm{DZ}$ as compared with $M Z$ pregnancies other than the increased range of fetal antigens not expressed in the mother. Zygosity is related to both maternal age and parity (Enders and Stern, 1948; Selvin, 1971). The MZ incidence remains relatively stable for age whereas $\mathrm{DZ}$ twins increase steadily up to about 38 years. Consequently a group of twin gestations in young women will contain a higher proportion of $M Z$ gestations. For example, in this series only 9 out of $28(32 \%)$ twin gestations in women under 20 years of age were unlike-sex, whereas in the over 30 category 158 of $241(66 \%)$ were unlike-sex. As pre-eclampsia is commoner in older women this could contribute to a higher incidence in association with $\mathrm{DZ}$ twins.

Also possibly relevant is the greater tendency for acute hydramnios to develop in monochorionic gestations (Naeye, 1964) which are all MZ. There is a consequent increased risk of premature labour at a stage so early that pre-eclampsia would have been unlikely to be manifest. In this series $6.2 \%$ of presumed monochorionic twins were delivered at 30 weeks or less compared with $3.0 \%$ of presumed dichorionic. If these cases of very premature delivery are omitted from the calculations there is a small relative increase in the incidence of severe pre-eclampsia in presumed $\mathbf{M Z}$ gestations (for 'unlike-sex DZ' the figure increases from $11.2 \%$ to $11.5 \%$, for 'presumed $M Z$ ' from $11.2 \%$ to $12.0 \%$ ). In addition death in utero of one twin is commoner in monochorionic gestations (Benirschke and Driscoll, 1967), and if this occurred early in pregnancy it would presumably reduce the tendency for preeclampsia to develop. Furthermore the validity of Weinberg's differential method has been questioned on evidence that there is normally an excess of likesex DZ over unlike-sex DZ twins (James, 1971). This would lessen the accuracy of comparing unlike- sex with like-sex twins and 'estimated' MZ twins. Cameron (1968) found $28 \%$ of his total series were $\mathrm{MZ}$ as judged by blood groups, enzyme studies, and placental examinations. Applying his figure to our data would give a total of $293 \mathrm{MZ}$ rather than the 349 twins estimated by Weinberg's method and $42 \%$ of the same sex twins would be $M Z$ rather than $50.1 \%$.

The accuracy of diagnosis of twins as di- or monochorionic from examination of single placentae at delivery is open to error (Nylander, 1970). In this series there were 65 placentae initially described as monochorionic for which a consultant pathologist's opinion was also available, supported where necessary by membrane histology. He found $53(82 \%)$ were monochorionic and 12 dichorionic. In Cameron's series $18 \%$ like-sex dichorionic twins were $M Z$. Applying the rate of error to the remaining 173 cases and allowing for this proportion of the dichorionic being $M Z$ then approximately $89 \%$ of the total 240 'monochorionic placentae' would be $\mathrm{MZ}$.

It has been suggested (Salzmann, 1955; Toivanen and Hirvonen, 1970) that toxaemia is commoner when there is a male fetus. This is relevant, for $75 \%$ of $\mathrm{DZ}$ twin pregnancies will have at least one male fetus but only $50 \% \mathrm{MZ}$ twin gestations will be male. Toassess this, twin pregnancies in which one or both babies were male were compared with pregnancies in which both babies were female. No significant difference was found in any category though in each the percentage incidence was marginally higher in the cases in which both twins were female.

It is interesting that Papiernik et al (1974) have observed a difference in weight gain in $\mathrm{DZ}$ as opposed to $\mathrm{MZ}$ pregnancies; $\mathrm{DZ}$ being associated with a greater gain. They attribute this to an ovular factor influencing the maternal weight change and explicitly state that it is not related to toxaemia incidence.

The matched-pair comparison with singletons showed an incidence of pre-eclampsia/eclampsia three times greater in twin pregnancies. This is probably an underestimate of the true difference as premature deliveries occur frequently because of the twin state itself, while in singletons a higher proportion of premature deliveries is attributable to in- 0 ductions of labour for severe pre-eclampsia, which $N$ would tend to bias the results contrary to the ob- N served difference. The incidence with twins was four times that with singletons if deliveries less than 36 weeks were excluded.

In $M Z$ twins the maternal-conceptus antigen differences are presumably the same as in singleton 7 pregnancies so, if such differences were the sole 
cause of the greatly increased incidence of preeclampsia in twins, they must be the result of the DZ twins which comprise about two-thirds of the total in Caucasian populations (Stevenson et al, 1971). Even if our data are ignored, it would be surprising if a threefold total increase could be the result of increased antigenic differences between mother and fetuses in two-thirds of the twins. If in a singleton pregnancy the conceptus antigens not expressed in the mother are $F$, it follows that the number of antigens in a $\mathrm{DZ}$ pregnancy not expressed in the mother would be $\angle 2 F$, and this maximum increase could only produce a rise in the pre-eclampsia/ eclampsia incidence of $<2 / 3 \times 2=<1.3$ while the observed factor is 3 .

\section{Conclusion}

While this study fails to show any consistent difference in the incidence of pre-eclampsia/eclampsia between $\mathrm{DZ}$ and $\mathrm{MZ}$ twin pregnancies, it does confirm the very greatly increased incidence of preeclampsia in association with twins-a threefold order compared with singletons. On a quantitative basis, considering numbers of antigen differences as a possible factor in causing pre-eclampsia, one would expect twin pregnancies to show an increase of $<1.3$. If total antigen 'dose' were involved it would be $<2$. The observed increase is greater than this particularly in the severe form of the disease. Furthermore, as indicated, the figures probably give an underestimate of the difference. Apparently the factor(s) responsible for the in- creased incidence of pre-eclampsia in twins do not represent a mere summation of those operative in two singleton pregnancies.

\section{REFERENCES}

Adams, E. M. and MacGillivray, I. (1961). Long term effect of pre-eclampsia in blood pressure. Lancet, 2, 1373-1375.

Benirschke, K. and Driscoll, S. G. (1967). The placenta of multiple pregnancy. In The Pathology of the Human Placenta, p. 165. Springer-Verlag, Berlin, Heidelberg New York.

Cameron, A. H. (1968). The Birmingham twin survey. Proceedings of the Royal Society of Medicine, 61, 229-234.

Enders, T. and Stern, C. (1948). The frequencies of twins, relative to age of mothers, in American populations. Genetics, 33, 263.

James, W. H. (1971). Excess of like-sexed pairs of dizygotic twins. Nature (London), 232, 277-278.

Kalmus, H. (1946). Genetical antigenic incompatibility as a possible cause of the toxaemias occurring late in pregnancy. Annals of Eugenics, 13, 146-149.

McCartney, C. P. (1964). Pathological anatomy of acute hypertension of pregnancy. Circulation, 29 and 30, Suppl. 11, 37-42.

Naeye, R. L. (1964). Fetal and neonatal development of twins. Pediatrics, 33, 546-553.

Nylander, P. P. S. (1970). The determination of zygosity-a study of 608 pairs born in Aberdeen. Fournal of Obstetrics and Gynaecology of the British Commonwealth, 77, 506-510.

Papiernik, E., Gerard, L., Hult, A. M., and Schneider, L. (1974). Hypothesis of an ovular regulation of pregnancy weight-gain. Acta Geneticae Medicae et Gemellologiae, 23, 56.

Penrose, L. S. (1946). On the familial appearances of maternal and fetal incompatibility. Annals of Eugenics, 13, 141-145.

Platt, R., Stewart, A. E., and Emery, E. W. (1958). The aetiology, incidence and heredity of pre-eclamptic toxaemia of pregnancy. Lancet, 1, 552-556.

Salzmann, K. D. (1955). Do transplacental hormones cause eclampsia? Lancet, 2, 953-956.

Selvin, S. (1971). The number of pregnancies prior to the birth of twins. Annals of Human Genetics, 34, 427-429.

Stevenson, A. C., Davison, B. C. C., Say, B., Ustuoplu, S., Lya, D., and Toppozoda, H. K. (1971). Contribution of fetal/maternal incompatibility to aetiology of pre-eclamptic toxaemia. Lancet, 2, 1286-1289.

Toivanen, P. and Hirvonen, T. (1970). Sex ratio of newborns : preponderance of males in toxaemia of pregnancy. Science, 170, 187188. 